\title{
PERCEPCIÓN DE LA CALIDAD DE VIDA EN EL ADULTO MAYOR QUE ACUDE A LA CLINIICA UNIVERSITARIA REFORMA
}

\begin{abstract}
PERSPECTIVE OF THE QUALITY OF LIFE IN THE ELDERLY WHO ATTEND THE CLINIC UNIVERSITARIA REFORMA
\end{abstract}

Recibido 14 de agosto 2017 Aceptado 20 de diciembre 2017

Correspondencia: Mtra. Elizabeth Araceli Izquierdo Barrera Correo:shamoyito19@gmail.com shamo_nequiz@hotmail.com

\section{Autores:}

Nequiz Jasso Jonathan Moisés

Pasante de la Lic. en Enfermería.

Facultad de Estudios Superiores Zaragoza.

Munguía Gómez Abraham

Pasante de la Lic, en Enfermería.

Facultad de Estudios Superiores Zaragoza.

Campus I Av. Guelatao No. 66 Col. Ejército de Oriente,

Mtra. Elizabeth Araceli Izquierdo Barrera

Facultad de Estudios Superiores Zaragoza. 
RESUMEN

Introducción: la calidad de vida con frecuencia es definida por la satisfacción que ocurre en el individuo contemplando aspectos ambientales, sociales, psicológicos y físicos. Para el adulto mayor, satisfacer cada uno de estos aspectos es importante y un tanto difícil principalmente por las circunstancias que se presentan en su vida cotidiana.

Objetivo: identificar la percepción de calidad de vida en el adulto mayor que acude a la clínica Universitaria Reforma.

Metodología: se realizó un estudio cualitativo, observacional, prolectivo, transversal y descriptivo, a 104 adultos mayores de 60 años, que acudieron a la Clínica Universitaria Reforma, en abril 2016. Se aplicó la escala de calidad de vida de la OMS WHOQOL y los datos obtenidos se analizaron a través de frecuencias y porcentajes.

Resultado: el 70\% de los adultos mayores tienen una percepción promedio de su calidad de vida; la cual incluyó 4 esferas: medio ambiente, con 74\%, salud física con 63\%, aspecto psicológico $62 \%$ y relaciones sociales 55\%.

Conclusión: las dimensiones que conforman la calidad de vida dependen de la percepción de las necesidades de la persona, a mayor satisfacción de éstas, mejor será su proceso de envejecimiento y por ende su calidad de vida será mejor.

Palabras clave: calidad de vida, adulto mayor, satisfacción.

\section{ABSTRACT}

Introduction: quality of life is often defined by the satisfaction that occurs in the individual contemplating environmental, social, psychological and physical aspects. For the older adult, satisfying each of these aspects is important and somewhat difficult, mainly due to the circumstances that arise in their daily life.

The objective of this study was to identify the perception of quality of life of the elderly who are treated at the Reforma University clinic.

Methodology: a qualitative, observational, prolective, cross-sectional and descriptive study was carried out on 104 adults over 60 years of age, who attended the Reforma University Clinic; in April 2016. The WHOQOL WHO quality of life scale was applied and the data obtained were analyzed through frequencies and percentages.

Result: $70 \%$ of older adults have an average perception of their quality of life; which included 4 spheres: environment, with $74 \%$, physical health with $63 \%$, psychological aspect $62 \%$ and social relations $55 \%$.

Conclusion: the dimensions that make up the quality of life depend on the perception of the needs of the person, the greater satisfaction of these, the better their aging process and therefore their quality of life will be better.

Key words: quality of life, older adult, satisfaction. 


\section{INTRODUCCIÓN}

L La Organización Mundial de la Salud (OMS) define la de calidad de vida, como la satisfacción que ocurre, en el individuo, de aspectos físicos y del medio ambiente. Para el adulto mayor percibir la satisfacción de cada uno de estos aspectos es importante para definir su calidad de vida, pues el equilibrio de la interacción de ellos en su vida cotidiana y el contexto en el que viven presenta barreras sociales y culturales que limitan su desarrollo integral. ${ }^{7-2}$

Según información de la Encuesta Intercensal 2015, en México hay 12.4 millones de personas de más de 60 años, lo que representa 10.4\% de la población total. La mayor parte de la población mayor de 60 (88.1\%) forma parte de un hogar familiar, mientras que $11.1 \%$ forman hogares unipersonales. Durante el segundo trimestre de 2016, la población ocupada de 60 y más años representa 33.6 por ciento.3.

Los adultos mayores, al igual que las personas jóvenes, tienen metas y actitudes que poseen aún durante esta etapa de la vida. Desgraciadamente, en México, se tiene una percepción muy negativa de la vejez, debido a que no se les visualiza de manera integral como personas con capacidades, intereses e inquietudes, ello provoca incluso una marginación laboral, familiar, social y política. La calidad de vida se define a partir de aspectos como la participación social, religiosa, cultural, económica, y afecto social, entre muchos más. ${ }^{4-5}$

La sociedad describe o percibe al adulto mayor de acuerdo a su experiencia; es por eso que para algunos la vejez es una etapa feliz y satisfactoria en la vida; pero otros más, pueden responder que es una etapa de sufrimiento, enfermedad, padecimiento, etc., además de las múltiples definiciones y descripciones que pueda haber de la vejez debemos considerar las familiares y sociales. ${ }^{6}$

Una de las principales formas para la valoración y estudio del adulto mayor, en todos sus aspectos; tanto psicológicos, sociales y biológicos, fueron las "teorías micro", primera generación de teorías de gerontología social que surgen de la interacción de la gerontología y la psicología social, razón por la cual tiene marcado un carácter individualista para el desarrollo del adulto, la cuestión esencial es la capacidad de interacción del individuo con su medio social y, para estudiarla en toda su extensión, se acude a factores de nivel micro como papeles, normas y grupos de referencia?

Pero no es suficiente llegar a la vejez para sentir la plenitud de la vida; su llegada no garantiza, por sí misma, su alcance. Por ello la vejez, dimensionada como fenómeno individual y colectivo, amerita una detención reflexiva y el riesgo de un preguntar sostenido acerca de su razón de ser en la vida humana. Al envejecer, la percepción de la propia imagen corporal, vida social y sexual se empieza a transformar, en este sentido, envejecer resulta más complicado para las mujeres por el gran peso cultural que se da a la juventud y a la maternidad. ${ }^{8}$

La importancia de la calidad de vida se debe ajustar a la esperanza de vida, de lo contrario aumentará la expectativa de incapacidad. Es necesario generar una mejor calidad de vida en el adulto mayor para incrementar el estado de confort en el aspecto social, para así poder mejorar el estadio de salud, la etapa funcional y un mejor equilibrio mental. Por lo que un adulto mayor que alcance cada vez más participación, expresión, relación, paz, dignidad y amor logrará una mayor calidad de vida. ${ }^{9}$

Se debe liberar al adulto mayor de esa imagen de incapacidad y soledad, contribuyendo a consolidar su papel social, redescubrir sus capacidades y habilidades, enriqueciendo su autoestima como protagonista activo. No se trata de afirmar que la población de edad mayor está aumentando, se requiere el incremento de espacios y actividades para ellos. ${ }^{10}$

El crecimiento de las tasas de población de adultos mayores ha suscitado el interés generalizado por un gran número de aspectos relacionados con las personas mayores. De entre ellos, tal vez la calidad 169 1 MNW Enf Neurol Vol. 16. No. 3 septiembre - diciembre 2017 
de la vida (CV) constituye, hoy por hoy, uno de los temas de estudio más importantes para las ciencias sociales y de la salud, pues supone una de las máximas aspiraciones de todo ser humano, quizás por ello se está convirtiendo en un tema frecuente tanto en la literatura científica, como en el uso que del tema hacen las personalidades sociales y políticas, tanto desde el punto de vista poblacional como del individual. 17,12

La presente investigación surge del interés en identificar la percepción de calidad de vida en adultos mayores que acuden a la Clínica Reforma, para analizar las representaciones que éste percibe en cuestión de su calidad de vida y las que enfrenta en su vida diaria.

\section{METODOLOGÍA}

巨 s un estudio de tipo cuantitativo, observacional, prolectivo, transversal y descriptivo, que se realizó a una población de 104 adultos mayores que acudieron, durante abril de 2016, a la Clínica Universitaria Reforma. Siendo la variable universal la percepción de la calidad de vida que el adulto mayor tiene durante esta etapa.

Se aplicó el instrumento WHOQOL-BREF de la OMS que consta de 26 preguntas; 24 de ellas están divididas en 4 dominios (físico, psicológico, relaciones sociales y medio ambiente) 2 preguntas son globales, cada una con 4 categorías tipo Likert para evaluar la calidad de vida del adulto mayor. El test proporciona un puntaje general relacionado con la percepción de la calidad de vida total.

El análisis de la información se realizó mediante estadística descriptiva utilizando el paquete SPSS (Statistical Package for the Social Sciences) versión 21.

\section{RESULTADOS}

La mayor parte de la población estuvo constituida por el sexo femenino 66\%, mientras que, el 34\% correspondía al sexo masculino; teniendo un rango de edad de 60 a 99 años, con una media de 70 años; se dividió en tres grupos por rango de edad, el primero de 60 -74 años representa un 70\%, el segundo de 75-84 años el 27\% y el grupo más longevo representa un 3\%. En cuanto al estado civil del adulto mayor el 56\% son casados; el 36\% son viudos; $3 \%$ son solteros; $3 \%$ separados; $1 \%$ divorciados y $1 \%$ en vive en unión libre con $1 \%$ respectivamente (tabla 1).

Tabla 1. Características sociodemográficas.

\begin{tabular}{|c|c|c|c|}
\hline $\begin{array}{r}\text { CARACTERÍS } \\
\text { DEMOG }\end{array}$ & $\begin{array}{l}\text { ICAS SOCIO- } \\
\text { RÁFICAS }\end{array}$ & $\begin{array}{c}\text { FRECUENCIA } \\
n=104\end{array}$ & $\begin{array}{c}\text { PORCENTAJE } \\
\%\end{array}$ \\
\hline \multirow[t]{3}{*}{ Sexo } & & ' & ' \\
\hline & Femenino & 69 & $66 \%$ \\
\hline & Masculino & 35 & $34 \%$ \\
\hline \multirow[t]{5}{*}{ Edad } & & ' & 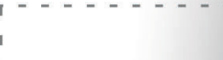 \\
\hline & $60-74$ & 73 & $70 \%$ \\
\hline & & ; & $27 \%$ \\
\hline & $15-84$ & 28 & 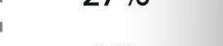 \\
\hline & $\geq 85$ & 3 & $3 \%$ \\
\hline \multicolumn{2}{|c|}{ Estado civil } & ; & 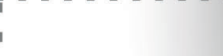 \\
\hline \multicolumn{2}{|r|}{ Soltero } & 3 & $3 \%$ \\
\hline \multirow{2}{*}{\multicolumn{2}{|c|}{ Separado }} & ; & $3 \%$ \\
\hline & & 3 & 610 \\
\hline \multicolumn{2}{|r|}{ Casado } & 58 & $56 \%$ \\
\hline \multicolumn{2}{|r|}{ Divorciado } & 1 & $1 \%$ \\
\hline \multicolumn{2}{|r|}{ Unión libre } & 1 & $1 \%$ \\
\hline \multicolumn{2}{|r|}{ Viudo } & 38 & $36 \%$ \\
\hline
\end{tabular}

Fuente: EGl, Abril 2016, Clínica Universitaria Reforma

En la evaluación de la percepción de calidad de vida, a partir de las 4 esferas: medio ambiente, aspecto psicológico, relaciones sociales y salud física, se observa el predominio de la calidad de vida promedio para las cuatro dimensiones, que en términos de favorable, el de medio ambiente es el de mayor importancia con $74 \%$, seguido de salud física con 63\%, aspecto psicológico 62\% y relaciones sociales 55\%. En la percepción de mala calidad se observa el dominio de la variable relaciones sociales con un $11 \%$, seguida en el medio ambiente con $8 \%$, salud física de $6 \%$ y aspectos psicológicos con 2\% (gráfica 1). 
Gráfica 1. Percepción de la calidad de vida

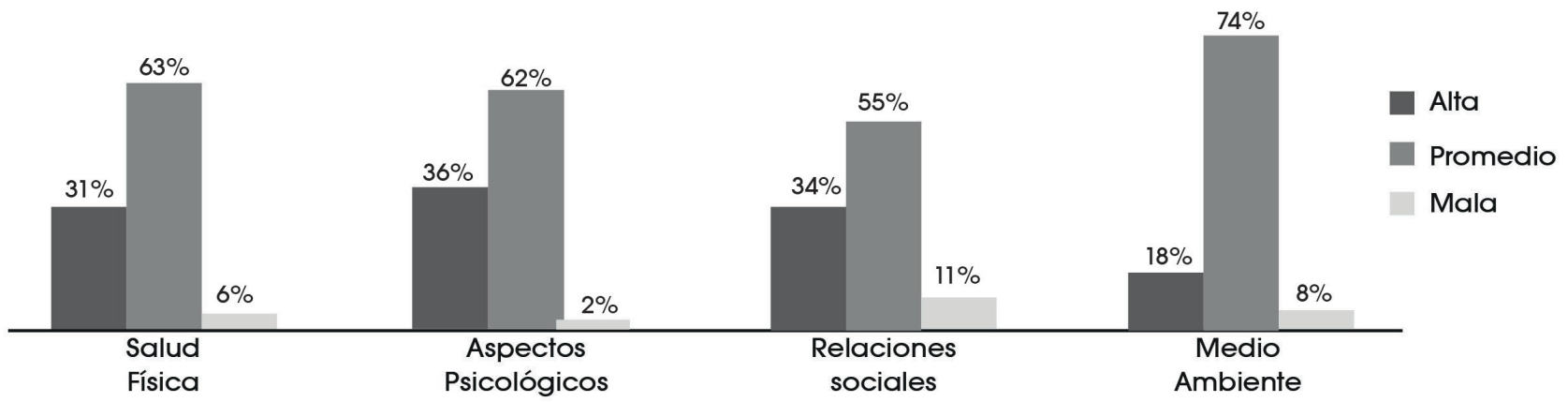

En relación a la percepción de la salúd física, 61\% de los adultos mayores consideran estar satisfechos con las habilidades que poseen, el 51\% considera tener la capacidad para trabajar y conciliar el sueño. Para El 38\% de ellos la energía que tienen es suficiente y, al igual que el 33\% piensan que posee la capacidad de movilidad necesaria para seguir realizando sus actividades diarias, sin sentir cansancio, el 30\% de ellos percibe que el dolor físico es una barrera para seguir con sus actividades de la vida diaria, ya que el 30\% ese mismo número de los adultos mayores complementa el tratamiento que llevan para cumplir con sus tareas o actividades sin problemas (gráfica 2).

Gráfica 2. Percepción de la salud física

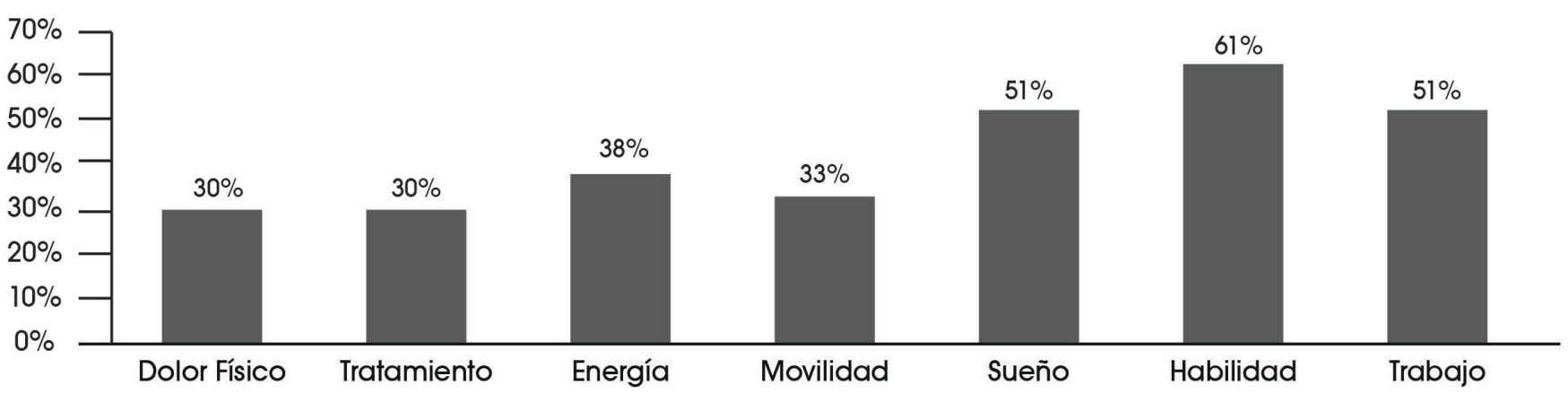

Del total de los adultos mayores se puede apreciar que el 63\% está satisfecho con su vida y el éxito que han alcanzado; sin embargo, no todos los adultos mayores disfrutan por completo de la etapa de la vejez pues sólo el 37\% refirió disfrutar de la vida moderadamente. Por otra parte, hallamos que sólo el 36\% encuentra un significado en su vida ya sea religioso, espiritual o personal; cabe destacar que la gran mayoría experimentan soledad, abandono y se refugian en grupos de apoyo que les ayudan a tener una vida feliz durante esta etapa. Esto se refleja en el hecho de que el 55\% de ellos refieren haber experimentado sentimientos de tristeza, desesperación, ansiedad o depresión. El 38\% logra concentrarse solo un poco en las actividades que realiza y, el 35\% acepta, forzosamente, su apariencia física actual, muchos añoran su aspecto físico cuando eran jóvenes (gráfica 3). 
Gráfica 3. Aspectos psicológicos

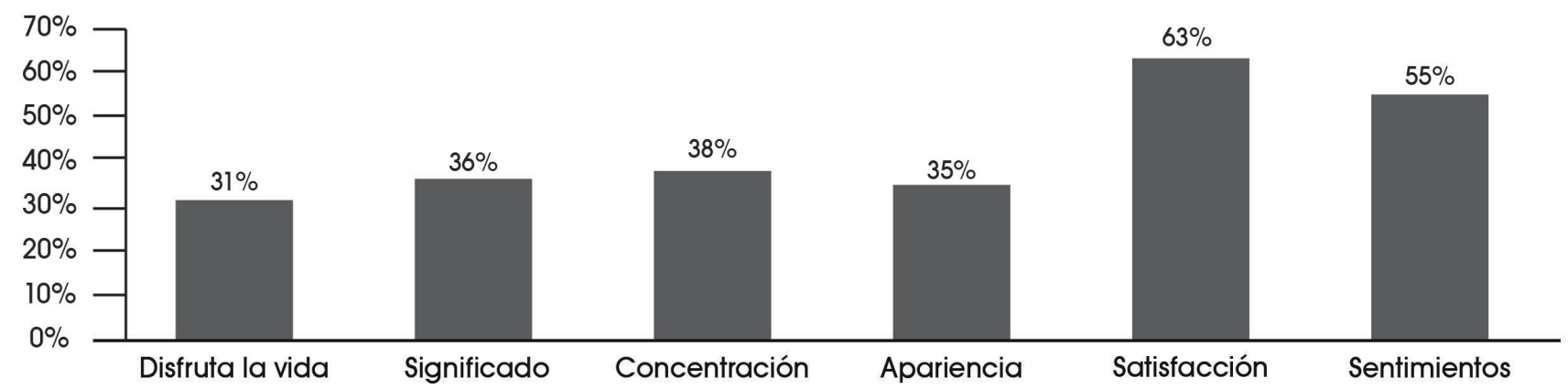

En relación a la satisfacción percibida en sus relaciones sociales, el 49\% de los adultos mayores están satisfechos con la relación que llevan con sus amigos, el 38\% de ellos encuentran satisfechos con su vida sexual, manifestando que la convivencia con su pareja es la adecuada. En cuanto a sus relaciones personales el 54\% están satisfechos (gráfica 4).

Gráfica 4. Percepción de la satisfacción social

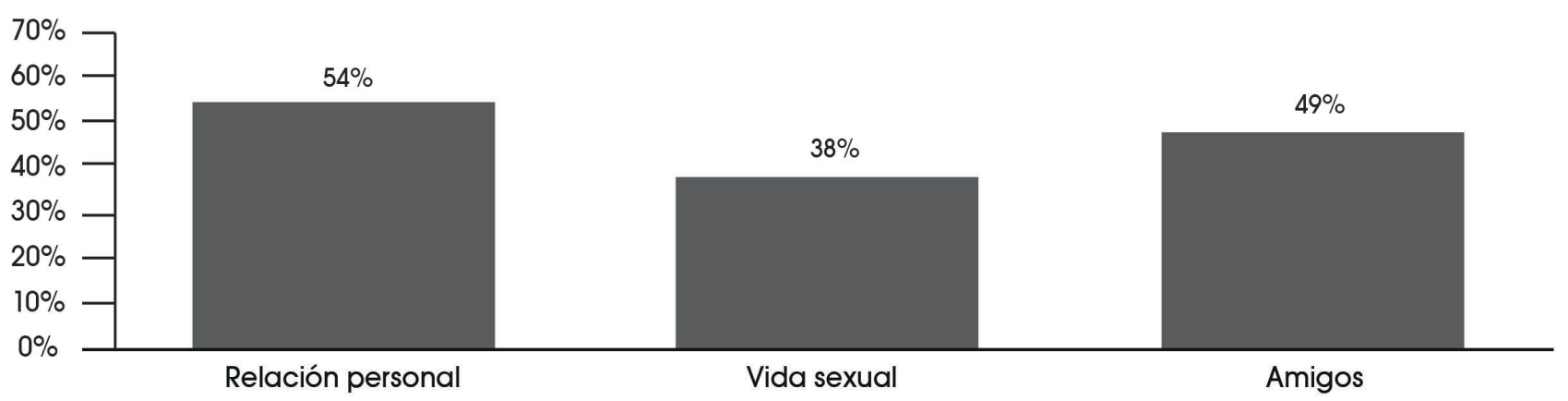

El 66\% se encuentra satisfecho, con su vivienda, para realizar sus actividades diarias; asimismo, el 59 \% refiere satisfacción con el acceso a los servicios de salud. El 51\% de ellos refiere que no logra cubrir todas sus necesidades básicas, de igual manera resulta con los medios de transporte e información, pues sólo el 38\% considera estar moderadamente satisfecho ya que, muchas veces, los transportes son ineficientes y no se encuentran adaptados con dispositivos de apoyo para los adultos mayores.
En cuanto al acceso a la información, con frecuencia los adultos mayores desconocen que hay apoyo para su desarrollo personal o los trámites para inscribirse en algún programa. En tanto a la participación en actividades recreativas el 37\% refieren no tener conocimiento de programas que les apoyen en este aspecto. Por lo que se refiere a seguridad, el 37\% menciona sentirse poco satisfecho y, el 49\%, considera estar medianamente complacido con el medio ambiente que los rodea (gráfica 5). 
Gráfica 5. Satisfacción de medio ambiente

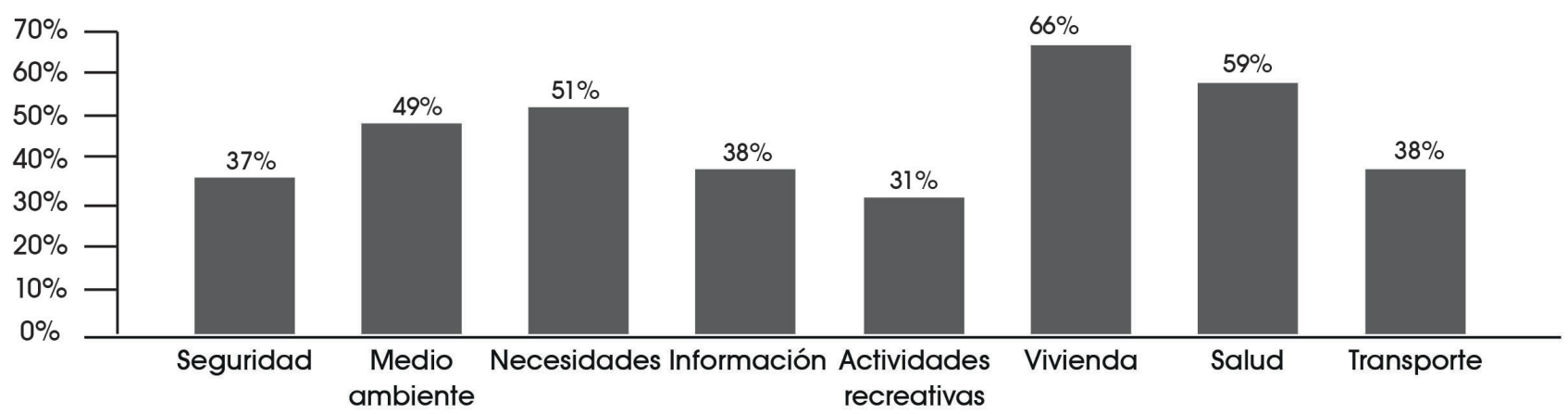

De forma individual, los adultos mayores evaluación, en promedio, es satisfactoria ya que encuestados evalúan su calidad de vida como implicaba su percepción sobre calidad de vida "ni pobre" y "ni buena" en un 46\% seguida de una a pesar de las condiciones físicas y psicológicas calidad de vida "buena" con 32\%. Es decir que la (gráfica 6).

Gráfica 6. Evaluación de calidad de vida

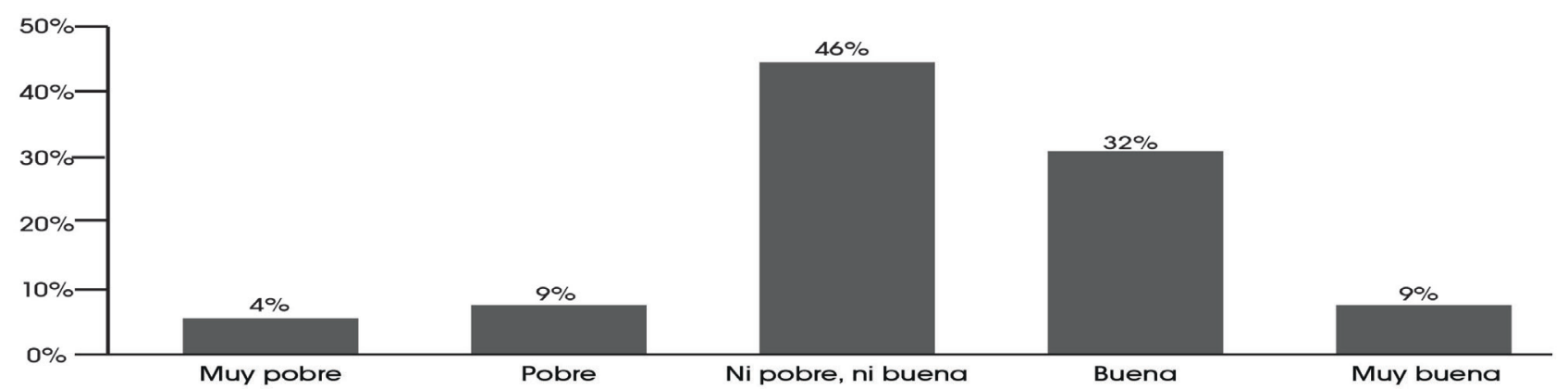

De forma general, la percepción de los adultos con un 70\%, seguida de alta con el 24\% y baja solo mayores, prevalece la calidad de vida promedio un 6\% (gráfica 7).

Gráfica 7. Calidad de vida

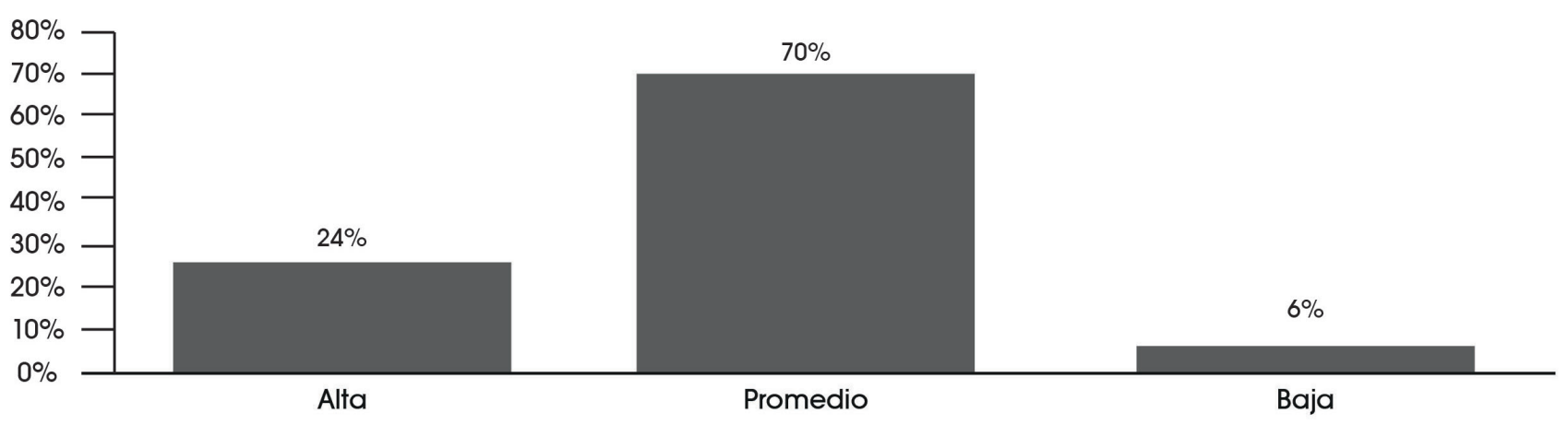




\section{DISCUSIÓN}

a percepción de calidad de vida que tienen los adultos mayores de la Clínica Universitaria Reforma se encuentra en un nivel promedio, en comparación con lo que menciona Cerón S. en el 201013 y Eugenia B. en 2007,14 donde se reporta que los adultos mayores tienen una buena y alta calidad de vida.

Los dominios por los cuales se define la percepción de calidad de vida, en este sentido se observa con una percepción promedio; en comparación con el estudio de González-Celis en su estudio calidad de vida en el adulto mayor del Instituto de Geriatría en México,15 donde se reportó que los adultos mayores entre 65 y 74 años, se concentran en tres dimensiones en particular: vitalidad, función social y salud mental que constituyeron los dominios en donde los adultos mayores tenían una percepción de calidad promedio.

Cabe resaltar que, en lo que respecta a las relaciones sociales, muchos refieren que la convivencia e, incluso la confianza, es mejor con sus amigos y su pareja que con la propia familia; ya que muchos son olvidados e incluso discriminados, volviendo mala la relación con los hijos, familia y sociedad.

La percepción del adulto mayor sobre su calidad de vida demuestra que la mayoría de la población pertenece a la categoría promedio; cabe mencionar que, aun con la falta de recursos económicos, capacidad para el trabajo y la no satisfacción en relación con familiares, se obtienen porcentajes altos de factores que no limitan a los adultos a encontrar satisfacción a su vida.

\section{CONCLUSIÓN}

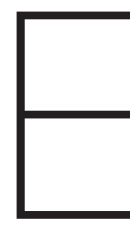

I objetivo fue conocer cómo percibe el adulto mayor su calidad de vida, a través del análisis de las representaciones sociales, psicológicas y biológicas que tiene de la misma. Este análisis permitió entender que la principal tarea de las personas mayores es la de comprender y aceptar su propia vida, utilizar su gran experiencia para hacer frente a los cambios personales o a sus pérdidas.

Se concluye que, en México el envejecimiento, para la mayoría de los pobladores, es un problema a largo plazo que afecta las expectativas que se han formado a lo largo de su vida, esto es producto con frecuencia que ante la sociedad el adulto mayor, da la imagen de ser una persona no útil y frágil dada su avanzada edad; de igual modo, la presencia de algunos trastornos y enfermedades. Para atacar está problemática es necesario formular estrategias que fomenten la participación activa y real de la población adulta mayor, en tanto personas que aportan desde sus saberes, necesidades y potencialidades. Es preciso asegurarnos que, para los adultos mayores se preserve y enriquezca la garantía de los derechos universales: salud, vivienda, alimentación, recreación, cuidado y participación, entre otros.

El envejecimiento de la población en México debe ser evaluada para con ello dar justa dimensión a sus defectos y virtudes, de manera que no sólo se eviten injusticias y discriminación. Pues, es imperante recordar que, los adultos mayores tienen la necesidad de plantearse nuevas metas y reorganizar su vida para evitar caer en la inutilidad y el ocio; en consecuencia con el tiempo, les puede provocar algún deterioro en la salud. Además, los profesionales de salud, tienen la obligación de velar por los adultos mayores para que los programas de promoción y prevención favorezcan su salud física, psicológica y social, y así, garantizar con ello su calidad de vida y apoyar al desarrollo satisfactorio de esta etapa. 


\section{REFERENCIAS BIBLIOGRÁFICAS}

1. Rubio-Olivares DY, Rivera-Martínez L, Borges-Oquendo L, GonzalesCrespo F. Calidad de vida en el adulto mayor. Varona (internet); 2015, juliodiciembre,no.67 (acceso 24-04-2016). Disponible en: http://www.redalyc.org/ pdf/3606/360643422019.pdf.

2. Flores M. Calidad de vida y salud en adultos mavores de viviendas multifamiliares en Guadalajara Jalisco, México. Procesos Psicológicos y Sociales, Revista Argentina de Clínica Psicológica (internet) 2010. Vol. 6 No. 1 y 2; (acceso: 26-04-2016). Disponible en: http://www.uacm.kirj.redalyc. redalyc. org/articulo.oo? id $=281937436010$

3. Instituto Nacional de Estadística y Geografía. (Internet). México: 2016 Estadísticas a propósito del día internacional de las personas de edad; (acceso 25- 04- 2017). Disponible en: http://www.inegi.org.mx/saladeprensa/ aproposito/2016/edad2016_0.pdf

4. Márquez, M.T. Transición. Envejecer no es de cobardes. México Editorial Norma: 2007.

5. Ham-Chande P. El envejecimiento: una nueva dimensión de la salud en México. Salud Pública (internet) 1996 Nov- Dic; vol. 38, No. 6; (acceso: 25- 042016). Disponible en: http://www.redalyc.org/articulo.oa?id=10638603

6. Arronte A, Beltrán C, Correa M, Martínez M, Mendoza N. Manual para la evaluación gerontológica integral en la comunidad, 2da Ed. México: FES Zaragoza, UNAM. 2008.

7. Díaz-Tendero A. Estudios de población y enfoques de gerontología social en México. Papeles de población (internet); vol. 17, núm. 70, octubrediciembre, 2011, pp. 49-79 Universidad Autónoma del Estado de México Toluca, México (acceso 24-04-2016) Disponible en: http://www.redalyc.org/ articulo.oa?id $=11221584004$

8. Arce H, Contreras P, Gutiérrez B. La vejez (conferencia). Madrid. Alfaguara Seminario Estudiantes de Psicología de la Universidad de Concepción. 2013
9. Schwartzmann L. Calidad de vida relacionada con la salud: aspectos conceptuales. Ciencias y Enfermería IX (Internet). 2003. octubre, Vol. 2 (acceso: 24-04-2016). Disponible en: http://www.scielo.cl/scielo php?script=sci_arttext\&pid=S0717-95532003000200002 00200002\&lng=es

10. Mendoza-Núñez VM, Martínez-Maldonado ML. Cambios biológicos durante el envejecimiento. En: Mendoza-Núñez VM, Martínez-Maldonado ML, Vargas-Guadarrama LA, editores. Gerontología Comunitaria. México FES Zaragoza, UNAM. 2004

11. Baster JC. Hacia un envejecimiento mayor. Correo científico médico de Holguin (internet); 2003 (editorial en línea). COCMED. 2004 marzo; Vol.7 No.2 Disponible en: http://www.cocmed.sld.cu/no72/n72edi.htm

12. Mendoza-Núñez VM, Martínez-Maldonado ML, Vargas-Guadarrama LA. Envejecimiento activo y saludable. Fundamentos y estrategias desde la gerontología comunitaria. México: FES Zaragoza, UNAM. 2013.

13. Cerón-Santes MI, Sanchez-Espinosa A, Riego-Azuara NA. Estudio sobre la percepción que tiene el adulto mayor de su calidad de vida. Portal médicos, (internet); 2013 (Acceso 28-04-2016) Disponible en: http://www. revista-portalesmedicos.com/revista-medica/percepcion-adulto-mayorcalidad-de-vida/

14. Botero E., Pico-Merchan M. Calidad de vida relacionada con la salud en adultos mayores de 60 años, hacia la promoción de la salud (internet') 2007 enero- dic, Vol. 12 (acceso: 24-04-2016.) Disponible en: http://www redalyc.org/articulo.oa?id=309126689002

15. González A.L, Rangel C. Calidad de vida en el adulto mayor, Instituto de Geriatría en México. (internet), 2009. (acceso 28-04-2016); Disponible en: http:// www.pactemospaz.utsem-morelos.edu.mx/files/librosdocentesydirectivos/ adultos_mayores/Calidad\%20de\%20Vida\%20Adulto\%20Mayor.pdf 